\title{
Telemedicine for Head and Neck Ambulatory Visits During COVID-19: Evaluating Usability and Patient Satisfaction
}

\author{
Karthik Rajasekaran, $\mathrm{MD}^{1}$ \\ ${ }^{1}$ University of Pennsylvania
}

May 11, 2020

\begin{abstract}
Background: In light of the COVID-19 pandemic, there has been a rapid increase in telemedicine visits. Otolaryngology patient satisfaction with these visits has not yet been extensively studied using a validated survey.

Methods: All patients who had telemedicine visits with three head and neck surgeons, by phone or video-based platform, between March 25, 2020 and April 24, 2020. Retrospective chart reviews were conducted to determine demographic, disease and treatment information. Patients who had a video visit were contacted by telephone and, if they could be reached and consented, were administered the Telehealth Usability Questionnaire (TUQ).

Results: 100 surveys were completed. The average score across all questions was 6.01 on a scale from 1-7, where 7 indicated the highest level of patient agreement. The highest scores were for questions related to satisfaction with telehealth (6.29), while the lowest were related to reliability (4.86).

Conclusions: Patients are generally highly satisfied with telemedicine.
\end{abstract}

Eleanor Layfield, $\mathrm{BA}^{1^{*}}$; Vasiliki Triantafillou, $\mathrm{MD}^{2^{*}}$; Aman Prasad, $\mathrm{BS}^{1}$; Jie Deng, PhD, RN, OCN, FAAN ${ }^{3}$; Rabie M. Shanti, DMD, MD' ${ }^{2}$; Jason G. Newman, MD ${ }^{2}$; Karthik Rajasekaran, $\mathrm{MD}^{2}$

${ }^{1}$ Perelman School of Medicine, University of Pennsylvania, Philadelphia, PA

${ }^{2}$ Department of Otorhinolaryngology-Head and Neck Surgery, University of Pennsylvania, Philadelphia, PA

${ }^{3}$ School of Nursing, University of Pennsylvania, Philadelphia, PA

${ }^{*}$ Denotes co-first authorship.

Address correspondence and reprint request to:

Karthik Rajasekaran, MD

Department of Otorhinolaryngology-Head and Neck Surgery

800 Walnut St, $18^{\text {th }}$ floor,

Philadelphia, PA 19107

215-829-5180

karthik.rajasekaran@pennmedicine.upenn.edu 
This work has not been printed or presented elsewhere.

Short title: Head and Neck Telemedicine Usability and Satisfaction COVID-19

Key words: telemedicine, COVID-19, Patient satisfaction, telehealth usability questionnaire, telehealth

Funding sources: The authors received no specific funding for this work.

Disclosure: The authors have no funding, financial relationships, or conflicts of interest to disclose.

\begin{abstract}
Background: In light of the COVID-19 pandemic, there has been a rapid increase in telemedicine visits. Otolaryngology patient satisfaction with these visits has not yet been extensively studied using a validated survey.

Methods: All patients who had telemedicine visits with three head and neck surgeons, by phone or videobased platform, between March 25, 2020 and April 24, 2020. Retrospective chart reviews were conducted to determine demographic, disease and treatment information. Patients who had a video visit were contacted by telephone and, if they could be reached and consented, were administered the Telehealth Usability Questionnaire (TUQ).

Results: 100 surveys were completed. The average score across all questions was 6.01 on a scale from 1-7, where 7 indicated the highest level of patient agreement. The highest scores were for questions related to satisfaction with telehealth (6.29), while the lowest were related to reliability (4.86).
\end{abstract}

Conclusions: Patients are generally highly satisfied with telemedicine.

\title{
Introduction
}

The COVID-19 pandemic is an unprecedented event that has led to an abrupt and drastic shift in the practice of medicine around the world. In an effort to balance the ongoing need for patient care with the risks to patient and staff safety, the CDC and multiple societies, including the American Academy of Otolaryngology - Head and Neck Surgery and the American Head and Neck Society, have issued recommendations to delay elective ambulatory provider visits or to consider telephone or video-based alternatives. ${ }^{1-3}$ In response, telemedicine has been rapidly implemented and widely adopted in many ambulatory practices for the first time.

The World Health Organization broadly defines telemedicine as the delivery of health care services by all health care professionals using technology for the exchange of valid information for diagnosis, treatment and prevention of disease and injuries. ${ }^{4}$ As an alternative to the traditional office encounter, telemedicine refers to a live (synchronous), two-way, interactive audio and video-based communication between the patient and the clinician to deliver care at a distance. While telemedicine has been successfully used in many fields over the past two decades, including some surgical subspecialties, ${ }^{5-7}$ its use has been less widespread in otolaryngology ${ }^{8}$ despite studies demonstrating that many otolaryngology diagnoses are amenable to virtual appointments ${ }^{9}$ and that telemedicine provides a significant cost savings and reduction in unnecessary office visits. ${ }^{10-12}$ This is in part due to difficulty of physical examinations in otolaryngology that often require equipment and training to perform. ${ }^{13}$

Prior to COVID-19, patient-facing telemedicine in otolaryngology has been largely limited to the delivery of care to remote areas, ${ }^{14}$ primary provider-to-specialist provider consultations ${ }^{10,12}$ or under specific circumstances, for instance in Navy military centers and shipboard medical departments ${ }^{15,16}$ or in the aftermath of 
Hurricane Katrina. ${ }^{17}$ Telemedicine in otolaryngology has less commonly focused on synchronous, interactive otolaryngology patient visits in general ${ }^{18-23}$ and few studies directly examine patient satisfaction. ${ }^{13,19-21,23}$

The sudden and sweeping transition to telemedicine in light of COVID-19 presents a unique opportunity to explore the role of telemedicine in otolaryngology ambulatory practices and patient satisfaction with virtual visits. Patient satisfaction is an important metric of healthcare quality and can play a major role in the long-term acceptance and success of a telemedicine program. As telehealth grows in prevalence and becomes further integrated into healthcare systems, it is important to preserve patient satisfaction across healthcare delivery modalities. Cursory examinations of patient satisfaction in prior studies have found high rates of patient satisfaction but these studies are limited by their small sample sizes and choice of patient satisfaction metrics. ${ }^{19-21,23}$ This study systematically explores patient satisfaction with video-based telemedicine visits during COVID-19 utilizing a validated telemedicine satisfaction survey in patients presenting to an academic head and neck surgery practice.

\section{Methods}

Intervention: Telemedicine Protocol

As part of the COVID-19 national emergency, the Office for Civil Rights (OCR) at the Department of Health and Human Services loosened the regulatory requirements under the Health Insurance and Portability and Accountability Act (HIPAA) to allow provider use of any non-public facing remote communication product to provide telehealth services. ${ }^{24}$ The earliest visits were conducted via Apple FaceTime, (Apple Inc., Cupertino, California, U.S.A.), keeping in line with the OCR's notification of enforcement discretion during COVID-19. Following department implementation of an institution-licensed platform, BlueJeans (BlueJeans, Verizon Enterprise Solutions LLC, Mountainview, California, U.S.A.), video-based visits were preferentially conducted through BlueJeans, whenever possible. Faculty received training in BlueJeans via virtual telehealth training sessions and recorded demos from the Penn Medicine Center for Connected Care, the University of Pennsylvania's telehealth center.

In the case where BlueJeans was unable to be utilized, video telemedicine visits were conducted by Doximity video dialer (beta, Doximity Inc., San Francisco, California, U.S.A.) accessible most easily on provider and patient smartphones or, as a last resort, Apple FaceTime (Apple Inc.). BlueJeans and Doximity both adhere to HIPAA recommendations with regard to security of protected health information. In all cases, patients were contacted by an administrative assistant from the office to assess capability for a video-based telemedicine visit, scheduled appropriately, and provided with instructions for accessing the visit. Just prior to the visit, a medical assistant called the patient via telephone to ask a series of intake questions and obtained verbal consent to conduct the video visit. The physician was then notified, and the visit was started. At the end of each video visit, the clinician stated that a research assistant would call to ask about the experience.

\section{Study Design}

This study was reviewed by the University of Pennsylvania Institutional Review Board (IRB) and was determined to be a quality improvement initiative that was exempted from further IRB review.

A retrospective chart review of telemedicine outpatient encounters by Head and Neck Otolaryngology faculty from March 25, 2020 to April 24, 2020 was conducted. All patients aged 18 years old or older seen via a telephone or video-based encounter were included. Demographic data was collected including (age, gender, race, and insurance status), and disease and treatment characteristics, and encounter type were collected from chart review.

Only patients with video-based telemedicine visits were contacted to complete the patient satisfaction survey and all participants provided verbal consent to participate (Figure 1). Patients answered demographic questions (education level) and questions regarding telemedicine visit characteristics (platform and device used, whether assistance was required for set up). A structured, likert-scale based survey consisting of the Telehealth Usability Questionnaire (TUQ) was administered. 
The Telehealth Usability Questionnaire (TUQ) was chosen to assess patient satisfaction with the video-based telemedicine visits based on review of the literature to identify validated tools. ${ }^{25}$ The TUQ is a comprehensive and validated survey tool that includes questions in the domains of usefulness, ease of use, interaction quality (effectiveness), reliability, and satisfaction. It was chosen for its ability to address changes in telehealth service delivery, for example the use of different platforms or devices, as well as its ability to measure the quality of the telemedicine interaction and patient satisfaction with the encounter. The TUQ was developed from existing telehealth questionnaires and has robust independent content validity and internal consistency evidence. $^{26}$

\section{Data Collection and Analysis}

Eligible patients were contacted by telephone, with the first attempt made on the same day as their telemedicine visit. Calls were made using Doximity dialer (Doximity Inc.) to allow for display of the hospital name on the patient's caller ID. Three attempts were made to contact the patient: after a nonanswered first phone call, two subsequent calls were made at different times and on consecutive business days prior to deeming the patient unable to be contacted. In the case of non-English speaking patients and laryngectomy patients, the survey was administered via caregivers who had also been present during the telemedicine visit. Demographic data for all patients and survey responses were anonymously collected and entered by three research assistants into a password-protected database.

Descriptive statistics were used to characterize the study population and summarize patient ratings of items on the TUQ according to the groupings proposed by the authors. ${ }^{26}$ Data were analyzed with Mann-Whitney $\mathrm{U}$ test. Statistical significance was set at $\mathrm{p}<0.05$. The design and findings of this study were written in accordance with SQUIRE 2.0 guidelines. ${ }^{27}$

The design and findings of this study were written in accordance with SQUIRE 2.0 guidelines. ${ }^{27}$

\section{Results}

\section{Patient Demographics}

145 patients had telehealth visits between March 18, 2020 and April 24, 2020. Telemedicine was used for both new and return patient visits including pre-operative discussions, post-operative visits, and oncologic surveillance.122 (84.1\%) were conducted using a video-based platform, while $23(15.9 \%)$ were conducted using audio alone. Common reasons why patients had audio-only visits included lack of access to necessary equipment, patient preference, and technical difficulties.

108 patients were successfully contacted and 100 (92.6\%) agreed to participate in the survey. Demographics of the patients can be seen in table 1. All patients were evaluated by one of the three head and neck cancer surgeons. Patient disease and treatment characteristics are presented in table 2. Patients most commonly used their smartphones (78\%), followed by their laptops (13\%) for the visit.

\section{Telehealth Usability Questionnaire}

The TUQ was used to assess the usability of this telehealth system with patients using a Likert scale to rate responses (1: strongly disagree; 2 : disagree; 3 : somewhat disagree; 4: neutral; 5: somewhat agree; 6 : agree; 7: strongly agree). The total average score was 6.01 . The highest scores were for questions related to satisfaction with telehealth (6.29), while the lowest were related to reliability (4.86). Average scores for all questions and subsections can be seen in table 3.

Patient Perception of COVID-19

Patients were additionally asked a single question about their perception of COVID-19. 100 patients responded to the statement "I am worried about my health during COVID." The average score, using the same scale from 1-7, was 5.03 (standard deviation 1.98). Patients who had finished oncologic treatment $(\mathrm{n}=54)$ reported an average score of 5.02, while patients awaiting or in the midst of treatment $(\mathrm{n}=20)$ had an average score of $5.90(\mathrm{p}=0.048)$. 


\section{Discussion}

Telemedicine has become increasingly important over the past several years and even more so during the COVID-19 pandemic. Telemedicine provides an option for providers and patients to connect in a way that limits possible exposure and conserves personal protective equipment. Without a vaccine candidate, SARSCoV-2 will likely continue to be a clinical concern even after the immediate pandemic ends. ${ }^{28}$ Otolaryngologists in particular are at increased risk for occupational exposure to the virus. ${ }^{29-31}$ It is thus important to consider the broader role telemedicine could fulfill in otolaryngology practices. However, it is equally important to examine how patients view their experience, as patient satisfaction is an important metric of healthcare quality and can play a major role in the long-term acceptance and success of a telemedicine program. ${ }^{32}$

Previous studies of patient satisfaction with telemedicine across medical specialties have shown that patients are generally satisfied. ${ }^{6,33}$ The few studies in the otolaryngology literature on patient satisfaction with telemedicine also demonstrated a high degree of satisfaction but are limited by small sample sizes and methodology, often using single measures of satisfaction, ${ }^{19,23}$ non-validated surveys,${ }^{20}$ or a selection of items from various satisfaction surveys without independent, final validation. ${ }^{21}$ To our knowledge, this is the largest study addressing patient satisfaction with telemedicine in otolaryngology and the only study utilizing a validated survey from the larger telemedicine literature. Moreover, this is the first study to examine otolaryngology head and neck patient satisfaction with telemedicine during the COVID-19 pandemic.

The majority of patients reported high satisfaction with telehealth visits, with average scores higher than 5 in the majority of components of the survey. This finding echoes the results of previous telemedicine patient satisfaction studies in otolaryngology. ${ }^{20,23}$ Specifically, patients noted that telemedicine increased access to healthcare services, saved time, and overall met their healthcare needs. Patients also frequently mentioned cost savings.

While the interface quality component of the survey generally received high scores across all three platforms, Item 10 (This system is able to do everything I would want it to be able to do) received one of the lowest averages scores with an average score of 5.27. Several patients who gave it a low score reported that they did so because telehealth visits do not allow for in-depth physical examinations including flexible laryngoscopy rather than considering the capabilities of the platform itself. On this topic, some patients noted that the ability to screen share through one of the platforms (BlueJeans) was an asset in that it allowed the physician to show and explain imaging findings similarly to an in-person visit, a feature not available on the other two platforms. Despite this, the high scores in the three other questions of this component suggest that patients were generally satisfied with the interface quality. As patients complete virtual consultations across a variety of clinical circumstances, platforms, and specialists, patients will likely become aware of additional ways telemedicine addresses their needs. Careful needs analyses will be necessary in future studies to better capture this component as telemedicine evolves and patient familiarity with telemedicine capabilities increases.

Patients also reported high satisfaction with interaction quality, indicating that the telemedicine visit was effective for provider-patient interactions. Patients indicated that they were able to both easily talk to (average score 6.60, item 11) hear (average score 6.63, item 12), and see (average score 5.91, item 14) the physician. This stands in contrast to previous work that has found the patient's ability to clearly hear the clinician is often poor. ${ }^{33,34}$ Notably, the most commonly reported issue with the telemedicine visits was an issue with audio. Once troubleshooted and resolved, patients highly rated the quality of the audio component. In 4 cases, poor interaction quality secondary to issues with connectivity or technical difficulties resulted in the conversion of a video-based telemedicine visits to a telephone call.

The reliability subsection, designed to assess if the telemedicine visit is as reliable as in-person service, also had a low average score. Part of this score is explained by Item 15 (I think the visits provided over the telehealth system are the same as in-person visits), which had the lowest average score (4.02) of the survey. Many patients, after hearing this question added additional narrative comments that visits by telemedicine 
were not the same due to limitations on physical exam and lack of "human touch". The other two questions of this section (Items 16 and 17) were commonly answered as not applicable, with many patients indicating that they had no issues or problems at all. Thus, the patients who did rate these statements were the ones with problems, contributing to the lower average score. Other studies have also found low scores on the reliability subsection. ${ }^{33,34}$ This could be explained by the same phenomena observed by the present study or related to other studies that introduced patients to new software that necessitated specific computers and fast internet speeds, which participants did not have. ${ }^{34}$ By contrast, patients in the present study used Facetime, a platform many routinely use, or relatively simple videoconferencing software on personal electronic devices.

Interestingly, no patients expressed doubts regarding security or quality of care provided by the telemedicine visit. Patients with high satisfaction with quality of care speculated that not all complaints would be appropriate for virtual consultation and that quality is thus circumstance dependent. This is in contrast to consumer market surveys that have demonstrated consumer concerns primarily around security of health information and the quality of care delivered through telemedicine. ${ }^{35}$ This shift in attitude could represent increasing patient utilization of and comfort with technology as it becomes more prevalent or may be secondary to increased acceptance of the necessity of virtual visits during COVID-19.

\section{Limitations}

The results of this study are certainly influenced by the social context and implications of the COVID-19 pandemic. An additional question administered to the patients allowed them to express concern about their health during COVID-19: this question had an average score of 5.03. As expected, patients with ongoing cancer treatment were more likely to worry about their health than those who had finished oncology treatment. In addition, many patients would qualify high scores by adding that they thought telehealth visits were appropriate in response to the ongoing pandemic.

Additional limitations of this study include bias in patient selection and response bias of those completing the survey. Only patients with video-based telemedicine visits were contacted to participate. Patients who did not have access to these platforms or the requisite technology and services were offered telephone calls with audio only. Notably, successful use of a telemedicine platform requires a baseline knowledge of technology and access to a smartphone or home brand..$^{38}$ Thus, this study did not capture the results of patients who due to socioeconomic circumstances or low technology literacy were unable to have a video-based telemedicine visit. This was the case for 23 patients among 145 total patients who completed a telemedicine over the course of one month. This study also suffered from some of the inherent issues with telemedicine research. Generally, patients report high levels of satisfaction with the healthcare they receive. ${ }^{25}$ The majority of patients in this study have established relationships with their physicians and thus could have had trouble separating their satisfaction from their care in general with their satisfaction pertaining to the telehealth visit. To limit this, the purpose of the study was explained and this distinction was explicitly addressed prior to administration of the survey.

\section{Future Directions}

The challenges in patient care created by COVID-19 have enabled a more rapid and ubiquitous integration of telemedicine into medical practice than ever before. The increased emphasis on telemedicine visits will likely permanently shape the future of medicine due to increasing familiarity with telemedicine by both patients and providers as a result of COVID-19. Many patients in the present survey stated they would continue to use telemedicine even after a return to some semblance to normalcy under the conditions that the decision to conduct a visit via telemedicine is patient-centered. They expressed concern that access to inperson office visits for issues otherwise deemed appropriate for telemedicine could be influenced by financial factors or insurance companies and that this would come at a cost to the doctor-patient relationship and patient satisfaction. As telemedicine becomes more engrained in medical practice, it will be necessary to transparently communicate with patients and maintain patient and provider discretion in the decision to conduct a telemedicine visit.

Furthermore, training and education in telemedicine will need to be augmented at all levels of medical 
education. Currently, telehealth training in medical school curricula is largely missing ${ }^{37}$ and there are limited guidelines for how to best conduct a telemedicine visit, specifically in otolaryngology. To address this, we previously published a set of practice guidelines for telemedicine for head and neck cancer patients. ${ }^{39}$

\section{Conclusion}

Telemedicine is an increasingly important option for ambulatory patient care during COVID-19 with implications for medical practice beyond the end of the pandemic. Telemedicine is an effective means to conduct a variety of outpatient counter types with high patient satisfaction, including new patient, return, post-operative, and oncologic follow-up visits in a head and neck otolaryngology practice.

\section{References}

1. Prepare to Care for COVID-19: Get Your Practice Ready. https://www.cdc.gov/coronavirus/2019ncov/hcp/preparedness-resources.html. Accessed April 28, 2020.

2. AAO-HNS Telemedicine Committee. Prioritizing Novel Approaches to Telehealth for All Practitioners. AAO-HNS. https://www.entnet.org/content/prioritizing-novel-approaches-telehealth-all-practitioners.

3. AHNS Leadership and Patient Care Division. How COVID-19 is Affecting our Head and Neck Community. AHNS COVID-19 Bulletin. https://www.ahns.info/covid-19-2020/.

4. Telematics WGC on H. A Health Telematics Policy in Support of WHO's Health-for-All Strategy for Global Health Development : Report of the WHO Group Consultation on Health Telematics . Geneva; 1998.

5. Huang EY, Knight S, Guetter CR, et al. Telemedicine and telementoring in the surgical specialties: A narrative review. Am J Surg . 2019;218(4):760-766. doi:10.1016/j.amjsurg.2019.07.018

6. Forbes RC, Solorzano CC, Concepcion BP. Surgical telemedicine here to stay: More support from a randomized controlled trial on postoperative surgery visits. Am J Surg . April 2020. doi:10.1016/j.amjsurg.2020.03.033

7. Gunter RL, Chouinard S, Fernandes-Taylor S, et al. Current Use of Telemedicine for Post-Discharge Surgical Care: A Systematic Review.J Am Coll Surg . 2016;222(5):915-927. doi:10.1016/j.jamcollsurg.2016.01.062

8. Garritano FG, Goldenberg D. Successful telemedicine programs in otolaryngology. Otolaryngol Clin North Am . 2011;44(6):1259-1274. doi:10.1016/j.otc.2011.08.003

9. McCool RR, Davies L. Where Does Telemedicine Fit into Otolaryngology? An Assessment of Telemedicine Eligibility among Otolaryngology Diagnoses. Otolaryngol - Head Neck Surg (United States) . 2018;158(4):641-644. doi:10.1177/0194599818757724

10. Kohlert S, Murphy P, Tse D, Liddy C, Afkham A, Keely E. Improving access to otolaryngologyhead and neck surgery expert advice through eConsultations. Laryngoscope . 2018;128(2):350-355. doi:10.1002/lary.26677

11. Philips R, Seim N, Matrka L, et al. Cost savings associated with an outpatient otolaryngology telemedicine clinic. Laryngoscope Investig Otolaryngol . 2019;4(2):234-240. doi:10.1002/lio2.244

12. Gilani S, Bommakanti K, Friedman L. Electronic Consults in Otolaryngology: A Pilot Study to Evaluate the Use, Content, and Outcomes in an Academic Health System. Ann Otol Rhinol Laryngol . 2020;129(2):170-174. doi:10.1177/0003489419882726

13. Holtel MR, Burgess LPA. Telemedicine in otolaryngology. Otolaryngol Clin North Am . 2002;35(6):12631281. doi:10.1016/S0030-6665(02)00095-6

14. Kokesh J, Ferguson AS, Patricoski C. Telehealth in Alaska: delivery of health care services from a specialist's perspective. Int J Circumpolar Health . 2004;63(4):387-400. doi:10.3402/ijch.v63i4.17756 
15. Haegen TW, Cupp CC, Hunsaker DH. Teleotolaryngology: A retrospective review at a military tertiary treatment facility. Otolaryngol - Head Neck Surg . 2004;130(5):511-518. doi:10.1016/j.otohns.2004.01.010

16. Melcer T, Hunsaker D, Crann B, Caola L, Deniston W. A prospective evaluation of ENT telemedicine in remote military populations seeking specialty care. Telemed $J$ E Health . 2002;8(3):301-311. doi:10.1089/15305620260353199

17. Arriaga MA, Nuss D, Scrantz K, et al. Telemedicine-assisted neurotology in post-Katrina Southeast Louisiana. Otol Neurotol . 2010;31(3):524-527. doi:10.1097/MAO.0b013e3181cdd69d

18. Ullah R, Gilliland D, Adams D. Otolaryngology consultations by real-time telemedicine. Ulster Med $J$ . 2002;71(1):26-29.

19. Yulzari R, Bretler S, Avraham Y, Sharabi-Nov A, Even-Tov E, Gilbey P. Mobile Technology-Based Real-Time Teleotolaryngology Care Facilitated by a Nonotolaryngologist Physician in an Adult Population. Ann Otol Rhinol Laryngol . 2018;127(1):46-50. doi:10.1177/0003489417745089

20. Rimmer RA, Christopher V, Falck A, et al. Telemedicine in otolaryngology outpatient setting - single Center Head and Neck Surgery experience. Laryngoscope . 2018;128(9):2072-2075. doi:10.1002/lary.27123

21. Seim NB, Philips RHW, Matrka LA, et al. Developing a synchronous otolaryngology telemedicine Clinic: Prospective study to assess fidelity and diagnostic concordance. Laryngoscope . 2018;128(5):10681074. doi:10.1002/lary.26929

22. Beswick DM, Vashi A, Song Y, et al. Consultation via telemedicine and access to operative care for patients with head and neck cancer in a Veterans Health Administration population. In: Head and Neck . Vol 38. John Wiley and Sons Inc.; 2016:925-929. doi:10.1002/hed.24386

23. Meng X, Dai Z, Hang C, Wang Y. Smartphone-enabled wireless otoscope-assisted online telemedicine during the COVID-19 outbreak.Am J Otolaryngol . April 2020:102476. doi:10.1016/j.amjoto.2020.102476

24. Office for Civil Rights/Department of Health and Human Resources. Notification of Enforcement Discretion for Telehealth. https:/www.hhs.gov/hipaa/for-professionals/special-topics/emergencypreparedness/notification-enforcement-discretion-telehealth/index.html. Accessed April 27, 2020.

25. Langbecker D, Caffery LJ, Gillespie N, Smith AC. Using survey methods in telehealth research: A practical guide. J Telemed Telecare . 2017;23(9):770-779. doi:10.1177/1357633X17721814

26. Parmanto B, Lewis, Jr. AN, Graham KM, Bertolet MH. Development of the Telehealth Usability Questionnaire (TUQ). Int J Telerehabilitation . 2016;8(1):3-10. doi:10.5195/ijt.2016.6196

27. Ogrinc G, Davies L, Goodman D, Batalden P, Davidoff F, Stevens D. SQUIRE 2.0 (Standards for QUality Improvement Reporting Excellence): Revised publication guidelines from a detailed consensus process. $B M J$ Qual Saf . 2016;25:986-992.

28. Xu S, Li Y. Beware of the second wave of COVID-19. Lancet . 2020;395(10233):1321-1322. doi:10.1016/S0140-6736(20)30845-X

29. Patel ZM, Fernandez-Miranda J, Hwang PH, et al. Precautions for Endoscopic Transnasal Skull Base Surgery During the COVID-19 Pandemic.Accept to Neurosurg .

30. Zou L, Ruan F, Huang M, et al. SARS-CoV-2 viral load in upper respiratory specimens of infected patients. N Engl J Med . 2020;382(12):1177-1179. doi:10.1056/NEJMc2001737

31. Givi B, Schiff BA, Chinn SB, et al. Safety Recommendations for Evaluation and Surgery of the Head and Neck during the COVID-19 Pandemic. JAMA Otolaryngol - Head Neck Surg . 2020. doi:10.1001/jamaoto.2020.0780

32. Kruse CS, Krowski N, Rodriguez B, Tran L, Vela J, Brooks M. Telehealth and patient satisfaction: A systematic review and narrative analysis. BMJ Open . 2017;7(8). doi:10.1136/bmjopen-2017-016242 
33. Serwe KM, Hersch GI, Pancheri K. Feasibility of Using Telehealth to Deliver the "Powerful Tools for Caregivers" Program. Int J Telerehabilitation . 2017;9(1):15-22. doi:10.5195/ijt.2017.6214

34. Faett BL, Brienza DM, Geyer MJ, Ped C, Hoffman LA. Enseñanza de las habilidades de autocontrol en personas con inflamación crónica de las extremidades inferiores y movilidad limitada: evidencia de la usabilidad de la telerehabilitación. Int J Telerehabilitation . 2013;55195(110):17-26. doi:10.5195/ijt.2013.6114

35. Consumers are ready for specialty virtual visits. Are you? | Advisory Board. https://www.advisory.com/research/market-innovation-center/resources/posters/are-you-ready-forspecialty-virtual-visits. Accessed April 28, 2020.

36. Masino C, Lam TCM. Choice of rating scale labels: Implication for minimizing patient satisfaction response ceiling effect in telemedicine surveys. Telemed e-Health . 2014;20(12):1150-1155. doi:10.1089/tmj.2013.0350

37. Waseh S, Dicker AP. Telemedicine training in undergraduate medical education: Mixed-methods review. $J$ Med Internet Res . 2019;21(4). doi:10.2196/12515

38. Rajasekaran, K. Access to Telemedicine - Are we doing all that we can during the COVID-19 Pandemic? Accepted to American Academy of Otolarygology-Head and Neck Surgery.

39. Prasad A, Brewster R, Newman J, Rajasekaran K. Optimizing your telemedicine visit during the COVID19 pandemic: Practice Guidelines for head and neck cancer patients [published online ahead of print, 2020 Apr 28]. Head Neck . April 2020. doi:10.1002/hed.26197

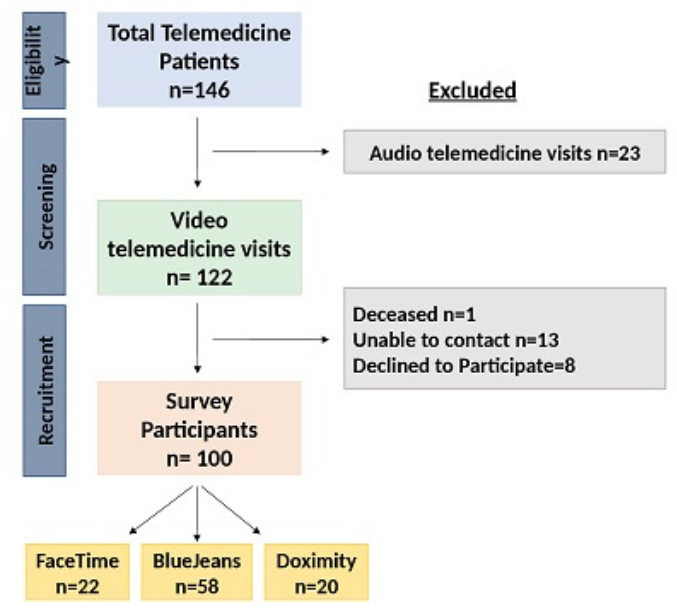

\section{Hosted file}

HED-20-0721_Table_1 (5).docx available at https://authorea.com/users/312328/articles/450167telemedicine-for-head-and-neck-ambulatory-visits-during-covid-19-evaluating-usabilityand-patient-satisfaction

\section{Hosted file}

HED-20-0721_Table_2 (3).docx available at https://authorea.com/users/312328/articles/450167telemedicine-for-head-and-neck-ambulatory-visits-during-covid-19-evaluating-usabilityand-patient-satisfaction

\section{Hosted file}


HED-20-0721_Table_3 (2).docx available at https://authorea.com/users/312328/articles/450167telemedicine-for-head-and-neck-ambulatory-visits-during-covid-19-evaluating-usabilityand-patient-satisfaction 